\title{
Functional analysis of the promoter of an early zygotic gene KLC2 in Aedes aegypti
}

\author{
Wanqi $\mathrm{Hu}^{1,2}$ and Zhijian Jake $\mathrm{Tu}^{1,2^{*}}$
}

\begin{abstract}
Background: Aedes aegypti is an important mosquito vector that transmits arboviruses that cause devastating diseases including Zika, dengue fever, yellow fever and chikungunya. Improved understanding of gene regulation in the early development of Ae. aegypti will facilitate genetic studies and help the development of novel control strategies of this important disease vector.

Results: In this study, we demonstrated through transgenic assays that the promoter of an endogenous early zygotic gene KLC2 could drive gene expression in the syncytial blastoderm and early cellular blastoderm, which is a stage that the developing germline and the rest of embryo are accessible to genetic manipulation. An unexpected expression of the reporter gene in transgenic male testes was also observed. Further analysis confirmed the expression of the endogenous KLC2 in the testes, which was not detected in the previous RNA sequencing data.
\end{abstract}

Conclusions: Our finding provided a new promoter element that can be used in future genetic studies and applications in Ae. aegypti. Moreover, our transgenic reporter assays showed that cautions are needed when interpreting RNA sequencing data as transient or tissue-specific transcription may go undetected by RNAseq.

Keywords: Early embryo, male, development, gene drive, vector, infectious disease

\section{Background}

Aedes aegypti is a major vector for Zika, dengue fever, yellow fever and chikungunya. These diseases affect tropical and subtropical regions worldwide, including Africa, the Americas, the Eastern Mediterranean, Southeast Asia and the Western Pacific. The rapid spread of Zika and the resulting microcephaly has caused major devastation in recent years. In the 2015 outbreak in Brazil, an estimated 440000-1300000 cases of Zika virus infection and over 4700 suspected cases of microcephaly have been reported $[1,2]$. The global incidence of dengue has increased dramatically in recent decades and nearly half of the world population is now at risk [3]. There are a few ongoing trials for vaccines. However, no specific treatment for dengue exists and current prevention depends solely on effective vector control [3], which is hindered by increasing insecticide-resistance [4-7]. Novel control strategies, informed by improved understanding of mosquito biology, are still needed. Population suppression and population

\footnotetext{
* Correspondence: jaketu@vt.edu

${ }^{1}$ Department of Biochemistry, Virginia Tech, 303 Fralin, Blacksburg, VA 24061, USA

${ }^{2}$ Fralin Life Science Institute, Virginia Tech, Blacksburg, VA 24061, USA
}

replacement are two such strategies, where genetic-modified mosquitoes are released to decrease the number or ability of mosquitoes transmitting diseases [8-13]. These methods require genetic manipulations and will benefit from precise control of transgene expression. For example, ectopic expression or knockdown of genes involved in sex-determination during early embryonic stages could produce genetic sexing strains, which are useful for population suppression approaches like the sterile insect technique [14-16]. To this end, a thorough knowledge of mosquito biology and gene regulatory elements is necessary.

Despite the great burden Ae. aegypti puts on public health, only a limited number of its promoter sequences have been described and tested in genomic context. Several promoters derived from endogenous genes are functional in midgut, fat body, salivary gland, ovary or testes [17-22]. Akbari and colleagues [23] reported 4 promoters which can drive strong maternal germline specific expression and be used for building a gene drive system termed MEDEA (Maternal Effect Dominate Embryonic Arrest [24]). No endogenous promoter that drives early zygotic gene expression has been tested in 
vivo in Ae. aegypti. Such a promoter is another major component of the MEDEA drive. In addition, early zygotic expression in the syncytial blastoderm and early cellular blastoderm affords the transgene or other factors access to the developing germline and the rest of embryo, which is beneficial to genetic manipulation. We previously sequenced staged Ae. aegypti embryos and found a list of genes that expressed at the onset of the syncytial blastoderm stage. We demonstrated that the 1 $\mathrm{kb}$ promoter region of one of the early zygotic gene (EZG) KLC2.1 was able to drive gene expression in early embryos through transient reporter assays [25]. In this study, we took a further step to establish the promoter activity in transgenic mosquitoes and showed that the 1 $\mathrm{kb}$ region of the KLC2.1 promoter initiate transgene expression very early in the embryos of Ae. aegypti. We also observed an unexpected transgene expression in testes and confirmed the same expression of the endogenous gene KLC2.1. Our study provides the first functional early zygotic promoter for future studies in genetic-modified mosquitoes or gene functions. Moreover, our results highlight an example in which tissue-specific expression of a gene may be missed in even relatively broadly sampled RNAseq data. In the most recent Ae. aegypti L5 genome assembly [26], KLC2.1 is merged with KLC2.2 and we will refer to this gene KLC2 hereafter.

\section{Results}

Generation and characterization of transgenic mosquitoes bearing the KLC2 promoter

Transgenic mosquitoes were created by co-injecting the transgene-containing donor plasmid and the transposasecontaining helper plasmid into the pre-blastoderm embryos of Ae aegypti Liverpool strain. The donor plasmid (Fig. 1a) contains a transformation marker, EGFP driven by the $3 \mathrm{xP} 3$ promoter, and a firefly luciferase coding cassette under the control of the $1 \mathrm{~kb}$ upstream sequence of KLC2. The transformation marker and the luciferase reporter cassette were flanked by piggyBac recognition sequence and were integrated into the mosquito genome in the presence of the piggyBac transposase provided by the helper plasmid. Seven transgenic lines were obtained from a single injection. All lines had characteristic EGFP expression in the eye with slightly different signal strength and expression patterns (Fig. 1b, Additional file 1). To eliminate the expression variance due to segregation of the heterozygous transgene in the progeny, we chose 1 of the 7 lines, JT0311-M3, to generate homozygous line after

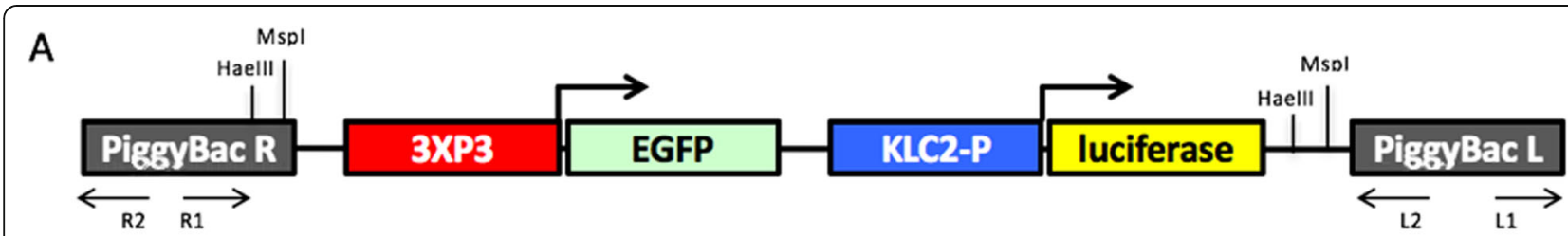

B
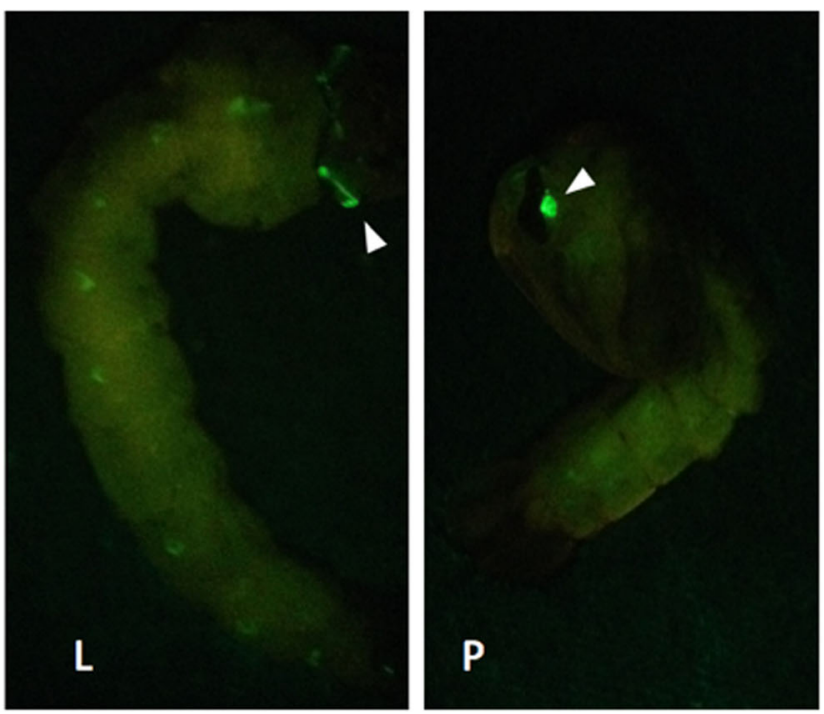

Fig. 1 The structure of donor plasmid used in embryo injection (a) and expression of the EGFP marker in transgenic mosquitoes (b). a The donor plasmid contains a transformation marker, EGFP driven by the 3xP3 promoter, and a firefly luciferase coding cassette under the control of the $1 \mathrm{~kb}$ upstream promoter sequence of KLC2 (KLC2-P). The entire sequence of the luciferase reporter cassette is provided as Additional file 6. piggyBac arms are also shown. Arrows indicate primer sets for inverse PCR (iPCR). Due to space limitation, nested iPCR primers are not drawn. All primers are shown in Additional file 3. $\mathbf{b}$ Images of transgenic positive larva $(\mathrm{L})$ and pupa $(\mathrm{P})$. Arrow heads point to EGFP marker expression in the eyes 
multiple generations of crossing and screening (thereafter refers to JT0311-M3-homo line). The Droplet Digital PCR (ddPCR) [27] confirmed that there was only one copy of the transgene in the JT0311-M3-homo genome (Fig. 2a) and the inverse PCR (iPCR) detected that the transgenes were reversely inserted in the intergenic region between AAEL009760 and AAEL009761 in the Supercont1.426 with at least $200 \mathrm{~kb}$ away from the neighboring genes (Fig. 2b, c).

The KLC2 promoter drives early zygotic expression of the luciferase transgene

We tested the KLC2 promoter activity by monitoring luciferase expression in different developmental stages. Consistent with the transcription profile of the endogenous KLC2 gene in early embryos [28], transient luciferase signals were observed in eggs from 3-5 h post-egg laying (PEL) to 7-9 h PEL (Additional file 2) in all 7 lines. A more detailed luciferase assay was performed using JT0311-M3-homo early embryos with 1-hour interval (Fig. 3a). A reverse transcriptase ddPCR (RT-ddPCR) was conducted to profile the transcripts in early embryos (Fig. 3b). The luciferase assay results, which illustrate luciferase protein abundance, and the RT-ddPCR results, which illustrate luciferase transcripts abundance, showed good correlations in early embryos
(Fig. 3a, b). Agreeing with the KLC2 expression profile [28], the luciferase gene was purely zygotic with no expression in $0-1 \mathrm{~h}$ and 1-2 $\mathrm{h}$ embryo. The transcription of luciferase gene started from 2-3 h PEL, peaked at 3-4 $\mathrm{h}$ PEL and dropped to background level after $6 \mathrm{~h}$ PEL. The measurement of luciferase protein showed approximate $1 \mathrm{~h}$ lag comparing with the transcripts, which started from 3-4 $\mathrm{h}$ embryos, peaked in 4-5 $\mathrm{h}$ embryos and disappeared in embryos older than $9 \mathrm{~h}$. The expression profile of luciferase gene perfectly mimicked the expression profile of endogenous KLC2 in early embryos (Biedler \& Tu, 2010,) (Additional file 3), indicating that the $1 \mathrm{~kb}$ upstream sequence of KLC2 was able to drive full expression in early embryos of Ae. aegypti.

\section{The KLC2 promoter also drives the expression of the transgene in testis}

While the expression of luciferase gene was predominant in early embryos, luciferase assays showed additional expression peaks in pupae and adult males in six of the seven heterozygous lines (Additional file 2). Because these seven lines were from different $G_{0}$ individuals and had slightly different EGFP patterns (Additional file 1), it is unlikely that they all had the same insertion site. All six lines had various levels of luciferase expression in pupae and adult males, indicating that this activity was

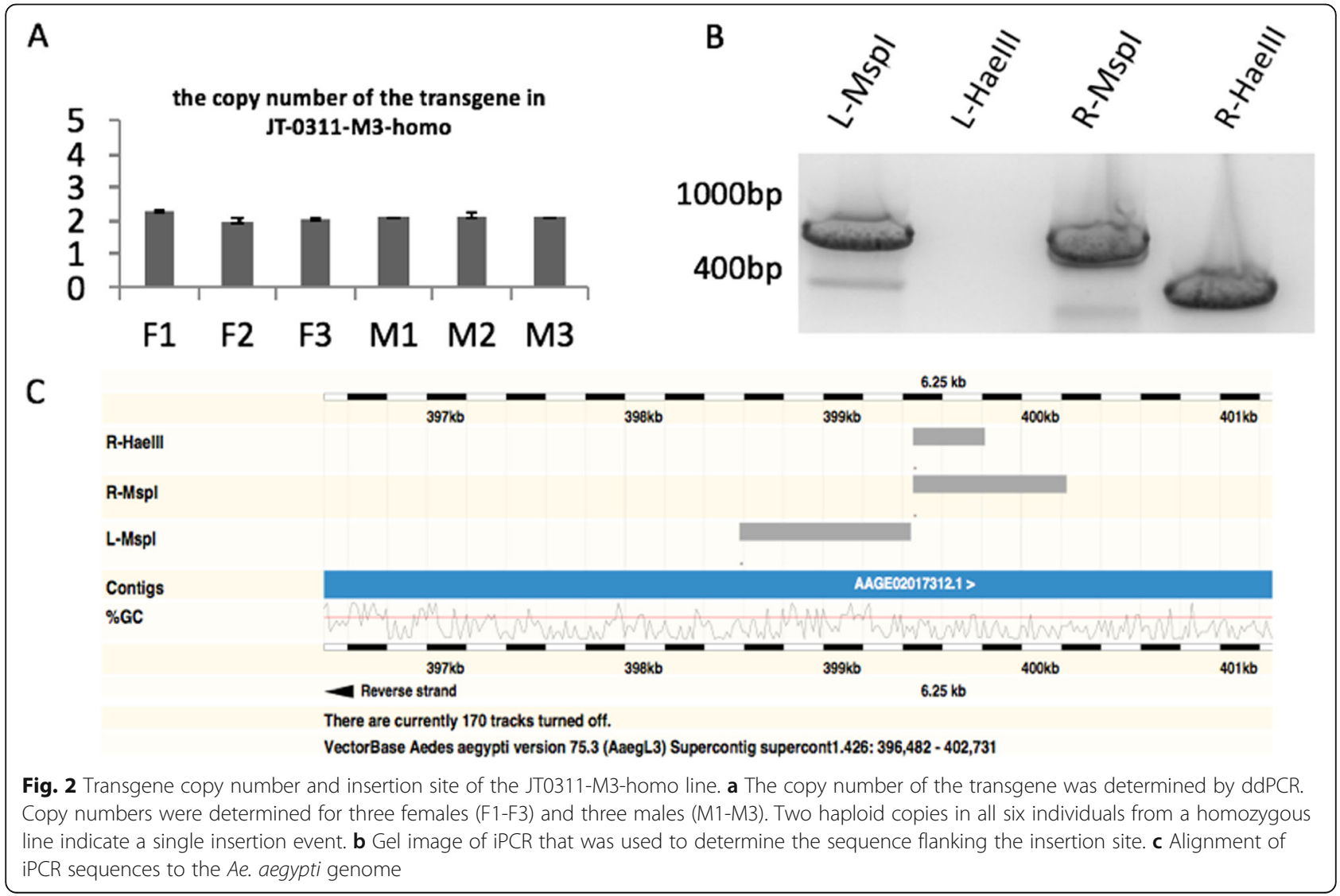




\section{A Luciferase assay (protein expression of luciferase)}

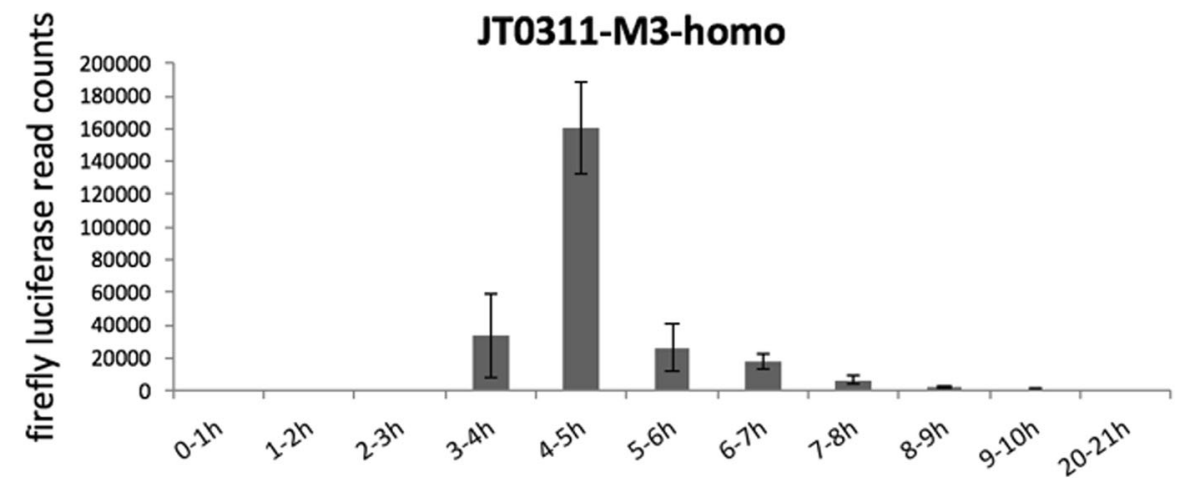

\section{B RT-ddPCR ( transcript level of luciferase)}

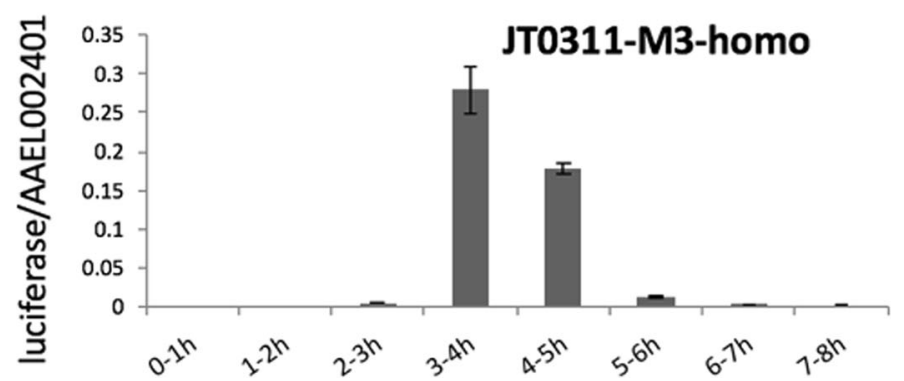

Fig. 3 Profiles of the luciferase protein activity (a) and transcript level (b) in the early embryos of the JT0311-M3-homo line. a The luciferase assay showed that the luciferase signal was first observed in 3-4 h embryos, peaked in the 4-5 h embryos and disappeared after 9-10 h. b The RTddPCR showed that the luciferase transcript was observed in 2-3 h embryos, peaked in 3-4 h embryos and disappeared after 7-8 h. AAEL002401 was used as an internal reference

not due to location effects. We therefore looked more carefully into the non-early-zygotic expression of luciferase in the JT0311-M3-homo line. Luciferase assays performed on sexed pupae showed that, like in the adult stage, the transgene was expressed only in male pupae (Fig. 4a). In the adult males, the luciferase signal was mainly from testes, as shown by the luciferase assay and RT-ddPCR (Fig. 4b, c). The male testis expression of the luciferase gene was unexpected because the endogenous KLC2 gene did not show male expression in previous RT-PCR or RNAseq analysis [28, 29] (Additional file 4). Nevertheless, when we did RT-PCR of the endogenous KLC2 on dissected male tissues, a band was observed in testis whereas no band could be detected when using male whole body as PCR template (Fig. 5).

\section{Discussion}

Comparing with model organisms such as Drosophila melanogaster, the knowledge and application of functional genetic elements in the yellow fever mosquito $A e$. aegypti are still quite limited. Often a tissue or temporal specific gene and its regulatory region were characterized first in Drosophila, and then their homologous counterparts were found and tested in mosquitoes [17, 20, 22, 30].
This transfer of knowledge does not always work given more than 200 million years of divergence between the two taxa. For example, the maternal and early zygotic promoters used to build the synthetic MEDEA element in Drosophila were derived from Drosophila genes bicoid and bottleneck that are not found in mosquitoes. The availability of the draft Ae. aegypti genome and the rapid development of next generation sequencing greatly facilitated the discovery of Ae. aegypti gene regulatory elements de novo. By applying transcriptome profiling, it is relative straightforward to find genes with expression in particular developmental stages or tissues. For example, Akbari et al. identified four germline promoters in Ae. aegypti through RNAseq recently [23]. In Anopheles stephensi, both maternal promoters and zygotic promoters have been tested using transgenic lines [31-33].

We previously found that KLC2 was a pure early zygotic gene with no maternal deposition and it is transcribed at the onset of the zygotic genome activation in Ae. Aegypti [28]. We chose the promoter of KLC2 for transgenic test based on a few observations. First, the expression of KLC2 was relatively high in early zygotic stage (ranked $13^{\text {th }}$ when sorted RPKM reads of all transcripts by ascending order in $0-2 \mathrm{~h}$ embryos and 


\section{A Luciferase assay}

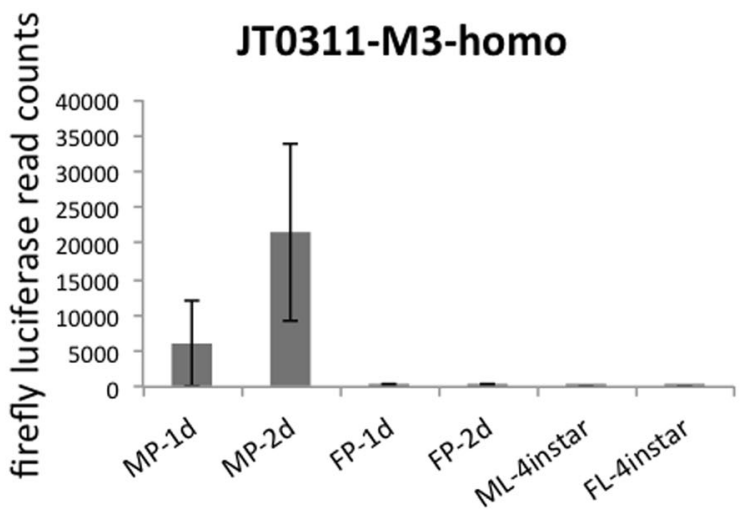

B

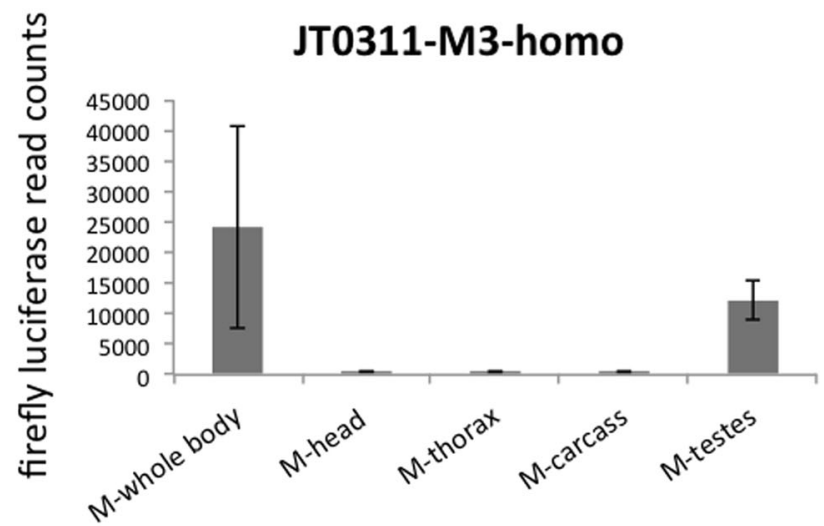

C RT-ddPCR

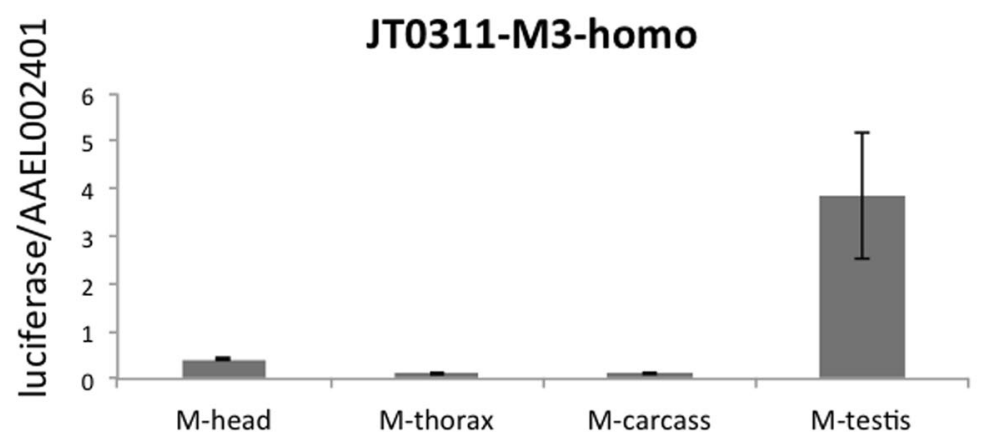

Fig. 4 Testis expression of the transgene. a The luciferase protein was detected in 1 and 2 day old male pupae (MP-1d, MP-2d) but not in 1 and 2 day old female pupae (FP-1d, FP-2d), male larvae (ML-4instar), or female larvae (FL-4instar), according to luciferase assay. b The luciferase protein activity in adult male was mainly detected in the testes. c RT-ddPCR showed that the luciferase transcripts were mainly detected in the male testes

descending order in 2-4 h embryos). Secondly, the expression profile of KLC2 was clean and only in early zygotic stage according to RNAseq. Thirdly, unlike other top-ranked early zygotic genes, KLC2 has fewer closely related paralogues and is relatively easy to study. Fourthly, transient luciferase assay demonstrated that the $1 \mathrm{~kb}$ upstream region of KLC2 was capable of driving gene expression in 5-6 h embryos $[25,28]$. In this study, we showed that the $1 \mathrm{~kb}$ promoter of KLC2 faithfully drove gene expression in transgenic Ae. aegypti early embryos. The transcription profile of the transgene in embryos was the same as that of endogenous KLC2 gene.

We also noticed a clear transgene expression in male pupae and adult testes. This male-specific activity was unlikely due to location of the transgene insertion sites because multiple lines bore the same expression pattern. Indeed, when we aligned the RNAseq data from two independent studies to the neighboring region of JT0311-M3-homo insertion site, we found no male-specific transcribed region [28, 29] (Additional file 5). The luciferase signal was barely detected in the $4^{\text {th }}$ instar male larvae, when spermatogonial mitosis begins to be observed [34]. This signal started to increase in young male pupae, when primary and secondary spermatocytes are generated through meiosis. The signal was highest in old male pupae and male adults, when most steps of spermatogenesis have completed [34]. It is interesting that in mouse, while the two major KLC

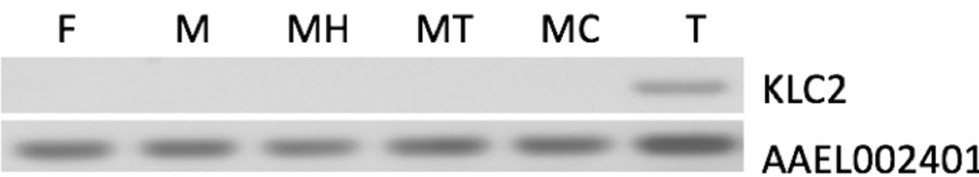

Fig. 5 RT-PCR showed that the endogenous KLC2 gene was expressed in testes. Lane F: female whole body; Lane M: male whole body; Lane MH: male head; Lane MT: male thorax; Lane MC: male carcass (abdomen without testis); Lane T: testis. AAEL002401 was used as the internal reference 
genes, KLC1 and KLC2 were not expressed in mature spermatids, another KLC gene, KLC3 was primarily expressed in post-meiotic male germ cells and was involved in sperm tail midpiece formation and sperm function [35, 36]. RT-PCR of dissected tissues using primers specific for Ae. aegypti KLC2 confirmed the expression of the KLC2 gene in adult testes. Taking together, our results suggest that the KLC2 may play a role in spermatogenesis or other testis-related functions in Ae. aegypti. However, functional analysis is needed to determine the precise role of KLC2 in male development.

We noticed that the testis transcription of the endogenous KLC2 was very weak while the expression of transgene was relatively high in testes. There could be another inhibiting element outside the $1 \mathrm{~kb}$ promoter region that suppresses testis expression of KLC2. Another explanation is that the stability of luciferase transcript and protein are higher than that of endogenous KLC2. The male expression of KLC2 was not detected in our RNAseq data $[25,28]$. This might be because we sequenced male whole bodies and the weak expression of KLC2 in testes was overwhelmed by transcripts in other parts of males. Indeed, RT-PCR did not show clear expression of KLC2 when male whole body was used. Curiously, RNAseq of $4 \mathrm{~d}$ male testes and accessory gland also failed to detect reads of KLC2 in an earlier study [29]. It is possible that the sequencing depth was not high enough to recover low abundant transcripts, or like in the early embryos, KLC2 may be transiently expressed in the testes, which could be missed during sample collection. Either scenario indicates the limitation of RNAseq in profiling genes that are transcribed in a narrow time window or in a small number of cells. Increasing sequencing depth and sample coverage will mitigate this problem.

\section{Conclusions}

The $1 \mathrm{~kb}$ promoter region of the KLC2 gene is able to drive gene expression at the beginning of the syncytial blastoderm stage in Ae. aegypti, making it a good resource for studying gene function during embryonic development and potentially for delivering transgene products to early embryos. For example, ectopic expression or knockdown of genes involved in sex-determination during early embryonic stages could produce genetic sexing strains, which are useful for population suppression approaches like the sterile insect technique [14-16]. Moreover, our transgenic reporter assays showed that cautions are needed when interpreting RNA sequencing data because transient and/or tissue-specific transcription may go undetected even in relatively broadly sampled datasets.

\section{Methods \\ Mosquitoes}

Aedes aegypti Liverpool strain (wild type) and all transgenic mosquitoes were reared at $28^{\circ} \mathrm{C}$ and $60 \%$ relative humidity on a $16 \mathrm{hr}$ light/8 hr dark photoperiod. Mosquito preblastoderm embryo injection and $\mathrm{G}_{0}$ transgenic mosquito screening were performed by the Insect Transformation Facility in Institute for Bioscience and Biotechnology Research at the University of Maryland (http://www.ibbr.umd.edu/facilities/itf). Microinjection into Ae. aegypti embryos was performed according to standard protocols [37] and the injection solution contains a $150 \mathrm{ng} / \mu \mathrm{l}$ donor plasmid (Fig. 1a, Additional file 6), a $300 \mathrm{ng} / \mu \mathrm{l}$ piggyBac transposase (phsp-Pbac) helper plasmid [38]. Third- or fourth-instar larvae of the transgenic lines were screened under fluorescent microscope for EGFP signal. All heterozygous lines were maintained by crossing transgenic positive males with wild type virgin females. To generate a homozygous line, heterozygous transgenic males and females were crossed. The positive offspring were then crossed with each other. When it was time to lay eggs, 20 females were allowed to lay eggs in individual tubes. The eggs were hatched separately, and larvae were inspected for any negatives. Any batches of larvae that appeared all positive were allowed to interbreed and the individual female egg collection was repeated. After several generations of inbreeding, the line was assumed homozygous when no negative larvae were observed.

\section{Copy number determination}

To determine the copy number of the transgene in the mosquito genome, ddPCR was performed on genomic DNA (gDNA) of JT0311-M3-homo mosquitoes using Bio-rad QX100 Droplet Digital PCR system following the previously published method [27]. Genomic DNAs were extracted from 3 adult male individuals and 3 adult female individuals by ZYMO Quick-gDNA Miniprep kit. Approximately 300-500 ng gDNA were digested with $D p n \mathrm{I}$ at $37^{\circ} \mathrm{C}$ for $1 \mathrm{~h}$ and used as ddPCR template after ethanol precipitation. $D p n I$ restriction digestion was performed to ensure separation of potential tandem repeats. A known single copy gene, nk homeobox protein AAEL006597, was used as an internal control. In all 6 tested-mosquitoes, two haploid copies were detected (Fig. 2a). Because JT0311-M3-homo is a homozygous line for transgene, this result indicated that there was a single insertion of the transgene. All primers and probes and ddPCR conditions are described in Additional file 2.

\section{Inverse PCR}

Approximately $1 \mu \mathrm{g}$ of JT0311-M3-homo gDNA was digested with either MspI or HaelII. After digestion, the 
product was ethanol precipitated and subjected to overnight ligation using $\mathrm{T} 4$ ligase at $4{ }^{\circ} \mathrm{C}$. The ligation product was again ethanol precipitated and used for PCR. Primers were designed to amplify either the left arm of the transgene plus genomic flanking sequences (primer set L1 and L2, Fig. 1a) or the right arm of transgene plus genomic flanking sequences (primer set R1 and R2, Fig. 1a). PCR products could be amplified from three of the four combinations (Fig. 2b). The bands were gel purified and sent for sequencing. The sequencing results were BLAST against Ae. aegypti genome. The BLAST output from left arm and right arm were compared to pin point the insertion site. Segments in supercont1.426 were the best hit in all three combinations, with left-arm flanking sequences aligning to supercont1.426: 398577-399439 and right-arm flanking sequences aligning to supercont1.426: 399453-399813 (HaeIII) or 400224 (MspI) (Fig. 2c). This location was the only location that showed a significant match in the BLAST output of all three inverse PCR sequences, confirming that there was a single insertion event. Primers and iPCR conditions are described in Additional file 2 and the approximate positions of the primers relative to the transformation donor plasmid are illustrated in Fig. 1.

\section{Luciferase assays}

Firefly luciferase assays were performed using reagents from Promega. Briefly, mosquito embryos or tissue samples were grounded in $50 \mu \mathrm{l}$ Passive Lysis Buffer. Twenty microliters of lysate were added into luciferase reagent $(1 \times$ luciferase substrate in buffer) and measured for luminescence immediately in GloMax 20/20 luminometer. We used 10 embryos, 1 larva, 1 pupa, 1 adult male or female whole body, 2 heads, 2 thoraces, 2 carcasses (abdomen without testes) or 2 pairs of testes for each measurement. The sex of pupae was differentiated by morphological structure [39]. The sex of larvae was decided by PCR of a male specific gene named myosex [40] on the gDNA extracted from a small amount of larvae tissue using a housekeeping gene (AAEL006597) as quality control. Primers and PCR conditions are described in Additional file 2.

\section{Reverse transcriptase PCR}

Total RNAs were extracted from staged embryos, adult males, adult females, male heads, thoraxes, carcass, or testes by ZYMO Quick-RNA MiniPrep kit. Approximately 20 ng RNAs were reverse transcribed to cDNA by Invitrogen SuperScript III RT kit. Regular RT-PCR and RT-ddPCR were performed on different cDNA samples using FAM-labeled probes targeting luciferase gene or endogenous KLC2 gene and Hex-labeled probe targeting an internal control housekeeping gene AAEL002401. The concentrations of target genes were normalized by dividing the concentration of AAEL002401 in each PCR well. All primers and probes and RT-PCR and RT-ddPCR conditions are described in Additional file 2.

\section{Additional files}

Additional file 1: Marker phenotypes of the seven transgenic lines. (XLSX $27 \mathrm{~kb}$ )

Additional file 2: Luciferase expression in seven independent transgenic lines. Firefly luciferase assays showed that except JT0311-F2, other transgenic lines all have two expression peaks during the mosquito life-cycle. The first one is in early embryos and the second one is in pupa or adult males. (PNG $99 \mathrm{~kb}$ )

Additional file 3: Primers and probes used in this study. (DOCX $19 \mathrm{~kb}$ )

Additional file 4: Expression profile of KLC2 (AAEL011410) based on RNA sequencing data adopted from (a) Biedler et al. [25] and (b) Akbari et al. [29]. Both RNAseq data showed pure early zygotic expression of KLC2 without expression in adult males or male testes. (PNG 95 kb)

Additional file 5: Alignment of RNAseq data [29] to the genomic region surrounding the insertion site. The grey bars in the top panel indicate the alignment of iPCR sequence to the genome, which enabled the identification of the insertion site. The mapping results of various RNAseq data showed that the genes in the surrounding region were not transcribed in only males. Thus, the observed testis-biased expression is unlikely caused by enhancers or promoters in the neighboring region. (PNG $93 \mathrm{~kb}$ )

Additional file 6: The sequence of the luciferase reporter cassette. (DOCX $116 \mathrm{~kb}$ )

\section{Abbreviations}

PEL: Post-egg laying; iPCR: Inverse PCR; ddPCR: Droplet digital PCR

\section{Acknowledgements}

This work was supported by NIH grants Al077680, Al113643, and Al123338 to ZT and by the Virginia Experimental Station. We thank Robert Harrell of the Insect Transgenic Facility at the University of Maryland for embryonic injections and for generating the transgenic lines, and Randy Saunders for mosquito care. This study benefitted from discussions at International Atomic Energy Agency funded meetings for the Coordinated Research Project "Exploring genetic, molecular, mechanical and behavioral methods of sex separation in mosquitoes.

\section{Funding}

This work was supported by NIH grants Al077680, Al113643, and Al123338 to ZT and by the Virginia Experimental Station. Publication costs for this study was provided by the International Atomic Energy Agency as part of the Coordinated Research Project "Exploring genetic, molecular, mechanical and behavioural methods of sex separation in mosquitoes"

\section{Availability of data and materials}

All data generated or analyzed during this study are included in this article.

\section{About this supplement}

This article has been published as part of Parasites \& Vectors Volume 11 Supplement 2, 2018: Exploring genetic molecular, mechanical and behavioural methods of sex separation in mosquitoes. The full contents of the supplement are available online at https://parasitesandvectors.biomedcentral.com/ articles/supplements/volume-11-supplement-2.

\section{Authors' contributions}

ZT and $\mathrm{WH}$ conceived the idea and designed the experiment; $\mathrm{WH}$ performed the experiment and data analysis. WH and ZT wrote the manuscript. All authors read an approved the final manuscript

Ethics approval and consent to participate Not applicable 


\section{Consent for publication}

Not applicable

\section{Competing interests}

The authors declare that they have no competing interests.

\section{Publisher's Note}

Springer Nature remains neutral with regard to jurisdictional claims in published maps and institutional affiliations.

\section{Published: 24 December 2018}

\section{References}

1. Bogoch II, Brady OJ, Kraemer MUG, German M, Creatore MI, Kulkarni MA, et al. Anticipating the international spread of Zika virus from Brazil. Lancet. 2016;387:335-6.

2. Victora CG, Schuler-Faccini L, Matijasevich A, Ribeiro E, Pessoa A, Barros FC Microcephaly in Brazil: how to interpret reported numbers? Lancet. 2016; 387:621-4.

3. Dengue and severe dengue [Internet]. World Health Organization. [cited 2018 Nov 14]. Available from: http://www.who.int/news-room/fact-sheets/ detail/dengue-and-severe-dengue

4. Grisales N, Poupardin R, Gomez S, Fonseca-Gonzalez I, Ranson H, Lenhart A. Temephos Resistance in Aedes aegypti in Colombia Compromises Dengue Vector Control. PLOS Negl Trop Dis. 2013;7:e2438.

5. Lima EP, Paiva MHS, de Araújo AP, da Silva EVG, da Silva UM, de Oliveira LN, et al. Insecticide resistance in Aedes aegypti populations from Ceará, Brazil. Parasit Vectors. 2011;4:5

6. Marcombe S, Mathieu RB, Pocquet N, Riaz M-A, Poupardin R, Sélior S, et al. Insecticide Resistance in the Dengue Vector Aedes aegypti from Martinique: Distribution, Mechanisms and Relations with Environmental Factors. PloS One. 2012;7:e30989.

7. Vontas J, Kioulos E, Pavlidi N, Morou E, della Torre A, Ranson H. Insecticide resistance in the major dengue vectors Aedes albopictus and Aedes aegypti. Pestic Biochem Physiol. 2012;104:126-31.

8. Akbari OS, Matzen KD, Marshall JM, Huang H, Ward CM, Hay BA. A synthetic gene drive system for local, reversible modification and suppression of insect populations. Curr Biol. 2013;23:671-7.

9. Hay BA, Chen $\mathrm{C}-\mathrm{H}$, Ward CM, Huang H, Su JT, Guo M. Engineering the genomes of wild insect populations: challenges, and opportunities provided by synthetic Medea selfish genetic elements. J Insect Physiol. 2010;56:1402-13.

10. Marshall JM, Pittman GW, Buchman AB, Hay BA. Semele: A Killer-Male, Rescue-Female System for Suppression and Replacement of Insect Disease Vector Populations. Genetics. 2011;187:535-51.

11. Wilke ABB, Marrelli MT. Genetic control of mosquitoes: population suppression strategies. Rev Inst Med Trop Sao Paulo. 2012;54:287-92.

12. Alphey L. Genetic control of mosquitoes. Annu Rev Entomol. 2014:59:205-24.

13. Adelman ZN, Tu Z. Control of Mosquito-Borne Infectious Diseases: Sex and Gene Drive. Trends Parasitol. 2016:32:219-29.

14. Gilles JRL, Schetelig MF, Scolari F, Marec F, Capurro ML, Franz G, et al. Towards mosquito sterile insect technique programmes: Exploring genetic molecular, mechanical and behavioural methods of sex separation in mosquitoes. Acta Tropica. 2014;132:S178-87.

15. Lees RS, Gilles JR, Hendrichs J, Vreysen MJ, Bourtzis K. Back to the future: the sterile insect technique against mosquito disease vectors. Curr Opin Insect Sci. 2015:10:156-62.

16. Bourtzis K, Lees RS, Hendrichs J, Vreysen MJB. More than one rabbit out of the hat: Radiation, transgenic and symbiont-based approaches for sustainable management of mosquito and tsetse fly populations. Acta Tropica. 2016;157:115-30

17. Adelman ZN, Jasinskiene N, Onal S, Juhn J, Ashikyan A, Salampessy M, et al. nanos gene control DNA mediates developmentally regulated transposition in the yellow fever mosquito Aedes aegypti. PNAS. 2007;104:9970-5.

18. Cho K-H, Cheon H-M, Kokoza V, Raikhel AS. Regulatory region of the vitellogenin receptor gene sufficient for high-level, germ line cell-specific ovarian expression in transgenic Aedes aegypti mosquitoes. Insect Biochem Mol Biol. 2006:36:273-81

19. Coates CJ, Jasinskiene N, Miyashiro L, James AA. Mariner transposition and transformation of the yellow fever mosquito, Aedes aegypti. Proc Natl Acad Sci U S A. 1998;95:3748-51.
20. Kokoza V, Ahmed A, Cho WL, Jasinskiene N, James AA, Raikhel A. Engineering blood meal-activated systemic immunity in the yellow fever mosquito, Aedes aegypti. Proc Natl Acad Sci USA. 2000;97:9144-9.

21. Moreira LA, Edwards MJ, Adhami F, Jasinskiene $N$, James AA, Jacobs-Lorena M. Robust gut-specific gene expression in transgenic Aedes aegypti mosquitoes. Proc Natl Acad Sci U S A. 2000;97:10895-8.

22. Smith RC, Walter MF, Hice RH, O'Brochta DA, Atkinson PW. Testis-specific expression of the beta2 tubulin promoter of Aedes aegypti and its application as a genetic sex-separation marker. Insect Mol Biol. 2007;16:61-71.

23. Akbari OS, Papathanos PA, Sandler JE, Kennedy K, Hay BA. Identification of germline transcriptional regulatory elements in Aedes aegypti. Sci Rep. 2014;4:3954.

24. Chen C-H, Huang $H$, Ward CM, Su JT, Schaeffer LV, Guo M, et al. A Synthetic Maternal-Effect Selfish Genetic Element Drives Population Replacement in Drosophila. Science. 2007:316:597-600.

25. Biedler JK, Hu W, Tae H, Tu Z. Identification of Early Zygotic Genes in the Yellow Fever Mosquito Aedes aegypti and Discovery of a Motif Involved in Early Zygotic Genome Activation. PloS One. 2012;7:e33933.

26. Matthews BJ, Dudchenko O, Kingan S, Koren S, Antoshechkin I, Crawford JE, et al. Improved Aedes aegypti mosquito reference genome assembly enables biological discovery and vector control. Nature. 2018;563:501-507. https://doi. org/10.1038/s41586-018-0692-z. Epub 14 Nov 2018. PMID:30429615.

27. Hindson BJ, Ness KD, Masquelier DA, Belgrader P, Heredia NJ, Makarewicz AJ, et al. High-throughput droplet digital PCR system for absolute quantitation of DNA copy number. Anal Chem. 2011;83:8604-10.

28. Biedler JK, Tu Z. Evolutionary analysis of the kinesin light chain genes in the yellow fever mosquito Aedes aegypti: gene duplication as a source for novel early zygotic genes. BMC Evol Biol. 2010;10:206.

29. Akbari OS, Antoshechkin I, Amrhein H, Williams B, Diloreto R, Sandler J, et al. The developmental transcriptome of the mosquito Aedes aegypti, an invasive species and major arbovirus vector. G3 (Bethesda). 2013;3:1493-509.

30. Anderson M a E, Gross TL, Myles KM, Adelman ZN. Validation of novel promoter sequences derived from two endogenous ubiquitin genes in transgenic Aedes aegypti. Insect Mol Biol. 2010;19:441-9.

31. Criscione F, Qi Y, Saunders R, Hall B, Tu Z. A unique Y gene in the Asian malaria mosquito Anopheles stephensi encodes a small lysine-rich protein and is transcribed at the onset of embryonic development. Insect Mol Biol. 2013;22:433-41

32. Criscione F, Qi Y, Tu Z. GUY1 confers complete female lethality and is a strong candidate for a male-determining factor in Anopheles stephensi. Yamashita YM, editor. eLife. 2016;5:e19281.

33. Biedler JK, Qi Y, Pledger D, Macias VM, James AA, Tu Z. Maternal GermlineSpecific Genes in the Asian Malaria Mosquito Anopheles stephensi: Characterization and Application for Disease Control. G3 Genes Genomes Genetics. 2015;5:157-66

34. Mescher SA, Rai KS. Spermatogenesis in Aedes aegypti. Mosquito News, 1966;26:45-51. https://archive.org/details/cbarchive_115468_ spermatogenesisinaedesaegypti1966.

35. Junco A, Bhullar B, Tarnasky HA, van der Hoorn FA. Kinesin Light-Chain KLC3 Expression in Testis Is Restricted to Spermatids. Biol Reprod. 2001:64:1320-30.

36. Zhang Y, Ou Y, Cheng M, Saadi HS, Thundathil JC, van der Hoorn FA. KLC3 is involved in sperm tail midpiece formation and sperm function. Dev Biol. 2012:366:101-10.

37. Lobo NF, Clayton JR, Fraser MJ, Kafatos FC, Collins FH. High efficiency germline transformation of mosquitoes. Nat Protoc. 2006:1:1312-7.

38. Handler AM, Harrell RA. Germline transformation of Drosophila melanogaste with the piggyBac transposon vector. Insect Mol Biol. 1999:8:449-57.

39. Mario vargas V. Sexual dimorphism of larvae and pupae of Aedes aegypti (Linn.). Mosquito News. 1968:28:374-9.

40. Hall AB, Timoshevskiy VA, Sharakhova MV, Jiang X, Basu S, Anderson MAE, et al. Insights into the preservation of the homomorphic sex-determining chromosome of Aedes aegypti from the discovery of a male-biased gene tightly linked to the M-locus. Genome Biol Evol. 2014;6:179-91. 